\title{
Captage et stockage d'énergie solaire dans l'habitat par le moyen de mur diode à chaleur latente
}

\author{
L. Bourdeau, A. Jaffrin et A. Moisan ${\left({ }^{*}\right)}^{*}$ \\ Laboratoire C.N.R.S. d'Ecothermique Solaire, Observatoire de Nice, France
}

(Reçu le 2 octobre 1979, accepté le 6 décembre 1979)

\begin{abstract}
Résumé. - L'application de l'énergie solaire au chauffage des locaux est facilitée par la mise au point récente de matériaux à changement de phase. Calculs et mesures montrent qu'une paroi à chaleur latente soumise au rayonnement solaire offre un meilleur rendement de captage et une meilleure stabilité dans la restitution qu'une paroi analogue sans changement de phase. Néanmoins, il est essentiel de réduire les pertes nocturnes, ce qui est fait dans le cas du mur diode par une circulation d'air en sens unique entre la face avant et le volume arrière isolé. Dans le cadre d'une circulation par convection forcée, une optimisation des conditions de captage et une étude de la cinétique des transferts thermiques au niveau du stockage ont été réalisées. En application, le bilan de fonctionnement d'un mur diode de $4 \mathrm{~m}^{2}$ soumis à des conditions naturelles a été dressé. Dans l'optique d'une extension du mur diode en un système entièrement passif, une étude préliminaire du fonctionnement en convection naturelle est présentée : des contraintes sévères sur les dimensions et les dispositions géométriques possibles d'une telle paroi sont mises en évidence.
\end{abstract}

\begin{abstract}
Solar energy applications to space heating can now take advantage of low temperature technics using recently developed phase change materials. Calculations and experiments show that a better collector efficiency and a smoother heat restitution are obtained in a collector-wall which contains a latent heat material compared to a similar wall with sensible heat only. It is however essential to reduce night heat losses, which is here achieved by separating the collector side from the storage side and organizing one-way convective heat transfers. In the case of forced convection, the collector efficiency was optimized by using a simple model; heat transfers in the storage were studied experimentally. As an application a $4 \mathrm{~m}^{2}$ diode wall was tested over the 1979 winter season in Nice. In the case of natural convection, where the diode wall operates passively, severe contraints are found on the possible dimensions and geometries of the system.
\end{abstract}

1. Introduction. - Le chauffage des locaux étant caractérisé par un niveau de température relativement bas, un tel secteur devrait être propice au développement de systèmes de captage solaire simples fonctionnant avec un bon rendement. Une difficulté se présente cependant au niveau du stockage thermique qui ne peut guère assurer une grande capacité, sur un faible écart de température, par l'utilisation de la seule chaleur spécifique des matériaux. La mise au point récente de divers matériaux changeant de phase au voisinage de la température ambiante et capable d'accumuler une quantité d'énergie calorifique allant de 50 à $70 \mathrm{kWh} / \mathrm{m}^{3}[1,2]$, sur un intervalle de température qui n'est plus limité que par la dimension des surfaces d'échange, modifie les données du problème.

$\left({ }^{*}\right)$ Escuela de ingeneria, I'niversidad de concepcion, Casilla 53 C Concepcion, Chili.
Dans la perspective de l'utilisation de l'énergie solaire à basse température, un objectif prometteur est la mise au point de parois d'habitat renfermant un stockage par chaleur latente : le volume de ces parois correspond bien aux besoins de stockage thermique à court terme, les surfaces d'échange, qui sont de l'ordre de grandeur des surfaces habitables, permettent des échanges suffisants avec un gradient de température de quelques degrés. La première solution qui s'impose par sa simplicité est celle d'un mur du type Trombe-Michel [3] dans lequel une chaleur latente remplace la chaleur sensible usuelle. Le chapitre 2 est consacré à cette solution.

Le paragraphe 2.1 montre d'un point de vue théorique l'a vantage qu'on peut tirer de cette version du mur Trombe. Le paragraphe 2.2 montre des résultats expérimentaux qui confirment cette situation. Néanmoins, on constate que les déperditions nocturnes sont d'un niveau inacceptable en l'absence 
d'isolation variable. C'est ce qui justifie la recherche d'une structure de paroi composite réalisant un effet de diode thermique grâce à la circulation en sens unique d'un fluide caloporteur entre une face captante et un volume stockeur isolés l'un de l'autre. La notion de mur diode à eau existe déjà [4] sous une forme de thermosiphon. Des systèmes à air fonctionnant en thermosiphon dus à $F$. Trombe mais sans stockage localisé existent également [5]. Le choix arrêté ici et décrit dans le chapitre 3 est celui d'un système à air en convection forcée entre une face captante et un volume de stockage faisant appel à un matériau à changement de phase (chliarolithe [1]), dont le point de fusion est de $28^{\circ} \mathrm{C}$ et la chaleur latente de $50 \mathrm{kWh} / \mathrm{m}^{3}$. Le paragraphe 3.2 décrit l'optimisation du fonctionnement du capteur grâce à un modèle mathématique simple. Le paragraphe 3.3 porte sur les échanges au niveau du stockage tels qu'ils ont été observés en laboratoire. Le paragraphe 3.4 rapporte les données expérimentales qui ont été extraites d'une campagne de mesures sur une réalisation en vraie grandeur soumise à des conditions naturelles. Enfin le chapitre 4 aborde les problèmes posés par la mise au point d'un mur diode passif fonctionnant en thermosiphon.

2. Murs Trombe à chaleur sensible et à chaleur latente. - Un mur Trombe est une masse à forte capacité calorifique et bonne conductivité disposée verticalement, face au sud, derrière un simple ou double vitrage et soumis au rayonnement solaire.

La chaleur récupérée est répartie en une fraction évacuée par convection naturelle vers le local habité et une fraction stockée par conduction dans le mur. On peut, par extension, ranger sous cette rubrique les murs d'eau (réservoirs empilés, cylindres verticaux) aussi bien que les murs classiques de béton, le mur Trombe à chaleur latente étant une autre extension évidente.

2.1 MODÉLISATION DU COMPORTEMENT DU MUR TROMBE. - Le but est de prédire les modifications apportées au comportement d'un mur Trombe classique par la substitution de matériaux à chaleur latente au béton.

Dans l'hypothèse simplificative d'un mur de dimensions infinies et d'épaisseur $e$, l'équation de la conduction s'écrit, pour un matériau homogène inerte :

$$
\rho C_{\rho} \frac{\partial T}{\partial t}=\lambda \frac{\partial^{2} T}{\partial x^{2}}
$$

où $T$ est la température, $t$ le temps et $\lambda / \rho C_{\rho}$ la diffusivité thermique. En présence de changement de phase mettant en jeu une chaleur latente de fusion $L$, la variation de la masse fondue s'écrit :

$$
L \frac{\partial y}{\partial t}=\lambda \frac{\partial^{2} T}{\partial x^{2}}
$$

Ces équations ont été traitées numériquement sur ordinateur Solar 16 par une méthode de différences finies, en tenant compte sur les faces extérieures du mur de la présence d'un matériau inerte (emballage PVC de 2,5 mm d'épaisseur), d'un apport radiatif sur la face sud correspondant à une journée type d'hiver, d'une masse d'air à $20^{\circ} \mathrm{C}$ au contact de la face nord du mur. La figure 1 montre l'évolution au cours de $24 \mathrm{~h}$ des différentes puissances thermiques (apports solaires, pertes vers l'avant, restitution vers l'arrière) qui prennent place au sein d'un mur de chaleur latente (chliarolithe) de 3,5 cm d'épaisseur et au sein d'un mur de béton de $15 \mathrm{~cm}$ d'épaisseur.

Le choix de l'épaisseur de chliarolithe est dicté par le montant des apports journaliers, le choix de $15 \mathrm{~cm}$ de béton est plus arbitraire : il correspond ici à un rendement de captage voisin pour les deux parois. Les conclusions déduites de cette figure restent valables pour des épaisseurs de béton plus grandes, telles que celles utilisées dans les murs Trombe usuels : le flux restitué au volume habitable par le mur à chaleur latente subit un déphasage moins grand par rapport aux gains solaires que celui restitué par le mur de béton; il est beaucoup plus étalé grâce au palier de solidification. Les pertes vers l'avant des deux murs sont voisines et représentent sensiblement $60 \%$ des apports solaires captés derrière une vitre simple. Ce pourcentage pourrait descendre à $45 \%$ en présence de deux vitres, mais les apports solaires

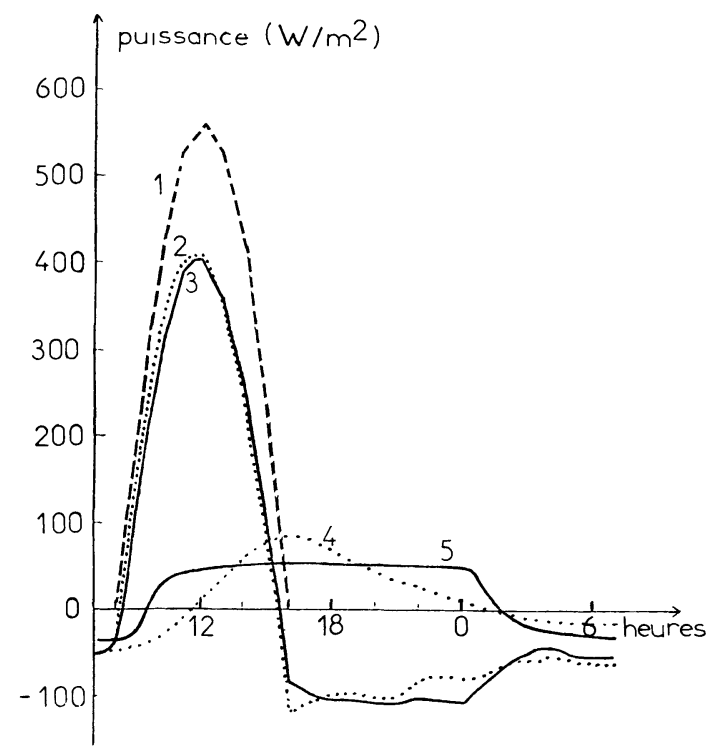

Fig. 1. - Résultat de modélisation des flux calorifiques transférés au cours d'une journée dans un mur Trombe en béton de $15 \mathrm{~cm}$ d'épaisseur (pointillés) et dans un mur à chaleur latente de $3,5 \mathrm{~cm}$ d'épaisseur (traits pleins). La courbe 1 désigne le flux solaire incident, les courbes 2 et 3 les flux échangés avec l'extérieur, les cou rbes 4 et 5 les flux échangés avec l'espace habitable.

[Numerical simulation of daily heat flux in a $15 \mathrm{~cm}$ thick concrete Trombe wall (dashed lines) and in a $3.5 \mathrm{~cm}$ thick latent heat wall (full lines). Curve 1 shows the incident solar radiation, curves 2 and 3 heat flux exchanged to the outside, curves 4 and 5 heat flux echanged with the living space.] 
seraient eux-mêmes réduits d'environ $10 \%$. En conclusion le bénéfice tiré de l'usage de chaleur latente dans un mur Trombe vient essentiellement d'un effet de régulation du transfert thermique vers l'habitat, mais les pertes nocturnes restent considérables et limitent le rendement global à une valeur voisine de $25 \%$ si l'on exclut le fonctionnement possible en thermosiphon du mur Trombe pendant la journée.

2.2 COMPARAISON EXPÉRIMENTALE D'UN MUR A CHALEUR LATENTE ET D'UN MUR A CHALEUR SENSIBLE. - Deux cellules tests ont été construites avec le but de soumettre une paroi de chaque type à un rayonnement et des températures d'hiver réalistes [6]. Chaque paroi de $3 \mathrm{~m}^{2}$ est constituée d'un réseau de tubes de PVC horizontaux de $5 \mathrm{~cm}$ de diamètre remplis dans un cas de chliarolithe et dans l'autre cas d'eau pure. Elles sont placées derrière un simple vitrage et constituent la paroi chauffante d'un local de $3 \mathrm{~m}^{3}$ isolé de l'extérieur par du polystyrène.

Les températures en plusieurs points du capteur, du stockage et du local chauffé furent enregistrées à la fin de l'hiver 1978. La figure 2 montre les températures observées dans les deux cellules tests lors d'une journée ensoleillée; si les courbes d'échauffement se ressemblent, à la pente près, les courbes de refroidissement montrent une différence très nette : contrairement à la décroissance hyperbolique observée pour le mur d'eau, les températures de la cellule à chaleur latente marquent un pallier de longue durée et l'amplitude des fluctuations sur $24 \mathrm{~h}$ est très réduite. Le bilan énergétique de cette journée montre
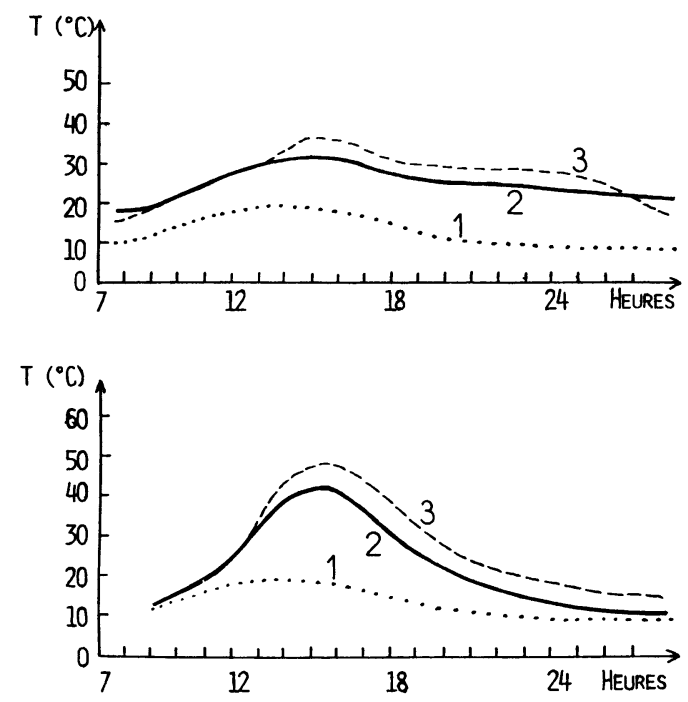

Fig. 2. - Comportement observé dans les cellules test au cours d'une journée. La figure $2 a$ montre le comportement de la cellule à chaleur latente, la figure $2 b$ le comportement de la cellule à eau. Les indices 1,2 et 3 se réfèrent respectivement à l'air extérieur, l'espace habitable et le stockage.

[Thermal behavior observed in the test cells on a typical dily. Figure $2 a$ is relative to the latent heat cell, and figure $2 b$ to the water cell. Indices 1,2 and 3 respectively refer to the exterior, the living space and the storage.] que le mur à chaleur latente a accumulé deux fois plus d'énergie que le mur d'eau. Son efficacité de captage a donc été double grâce à une température de surface plus faible. La température du local a pu être maintenue pendant la totalité de la nuit plus de $10^{\circ} \mathrm{C}$ au-dessus de la température extérieure. Néanmoins la constatation qui s'impose encore est que les pertes vers l'avant du mur sont dominantes.

Ces conclusions amènent naturellement à envisager une paroi plus complexe où les déperditions seraient plus faibles.

3. Mur diode à chaleur latente. - 3.1 STRUCTURE INTERNE. - Le mur diode est constitué d'une face captante séparé du volume de stockage par un isolant, le transfert de la chaleur se faisant par une boucle d'air en convection forcée. La figure 3 en montre la structure de principe. Le stockage contient le matériau à chaleur latente sous diverses formes possibles d'emballage, allant du cylindre de PVC à l'alvéole de béton. L'arrêt de la ventilation suffit à interrompre tout transfert de chaleur autre qu'une conduction résiduelle et un effet momentané de thermosiphon fortement réduit par les pertes de charge. Il est possible de prévoir un clapet antiretour.

3.2 MOdÉlisation DE LA FACE CAPTEUR A AIR. Il est important d'optimiser le fonctionnement du capteur selon la température d'air nécessaire au stockage, afin d'éviter une température d'absorbeur trop élevée.

Le modèle utilisé pour décrire les échanges au niveau du capteur à air en convection forcée est classique [7, 8] . Il consiste à supposer des valeurs constantes des températures de vitre $T_{\mathrm{v}}$ et d'absorbeur $T_{\mathrm{p}}$ en fonction de la position sur le capteur. Seule la température $\mathrm{du}$ fluide caloporteur est admise à varier le long de son parcours. Les échanges radiatifs entre vitre et capteur (par exemple) sont linéarisés en définissant un coefficient d'échange

$$
h_{\mathrm{rpv}}=\sigma\left(T_{\mathrm{p}}+T_{\mathrm{v}}\right)\left(T_{\mathrm{p}}^{2}+T_{\mathrm{v}}^{2}\right) /\left(1 / \varepsilon_{\rho}+1 / \varepsilon_{\mathrm{v}}-1\right)
$$

qui dépend de $T_{\mathrm{p}}$ et $T_{\mathrm{v}}$. On en déduit une loi exponentielle pour la température de fluide en fonction de sa position $y$, de la forme

$$
T_{\mathrm{f}}=T_{\mathrm{f}_{\infty}}-\left(T_{\mathrm{f}_{\infty}}-T_{\mathrm{fi}}\right) \exp -a y
$$

où $a$ dépend des coefficients de déperdition et du débit.

Cette expression permet à son tour de définir une température moyenne de fluide, si bien que toute la description des processus de transferts thermiques dans le capteur se fait au moyen de températures fixes, indépendantes de la position. Dans ces conditions, l'énergie récupérée par le fluide prend la forme

$$
Q=F\left[S-U_{1}\left(T_{\mathrm{f}}-T_{\mathrm{a}}\right)\right]
$$




\section{Circulation \\ air forcé}

Rayonnement solaire

\section{Circulation air forcé}

Ambiance extérieure

Vitrage sumple nu double

Surface absorrante Isolant thermique

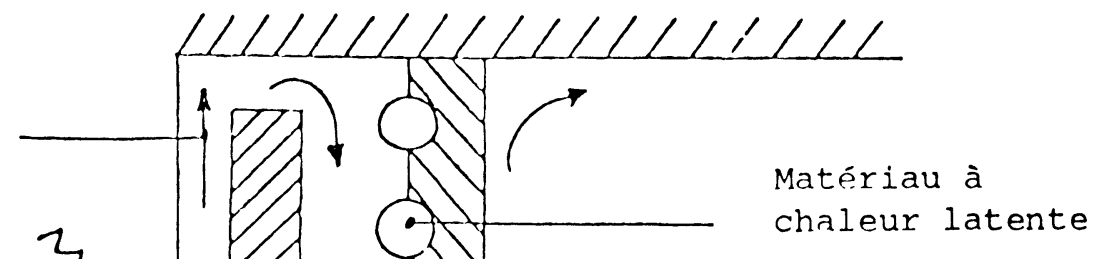

Mur du local

Local à chauffer

Rayonnement vers le local

Convection naturelle dans le local

Fig. 3. - Coupe schématiquement d'un mur diode à circulation d'air et à stockage par chaleur latente.

[Schematic view of latent heat diode wall with forced air convection.]

où

$$
F=\left[1+h_{\mathrm{rpr}} U_{\mathrm{t}} /\left(h_{\mathrm{rpr}} h_{\mathrm{ra}}+h_{\mathrm{pa}} U_{\mathrm{t}}+h_{\mathrm{pa}} h_{\mathrm{rpv}}+h_{\mathrm{va}} h_{\mathrm{pv}}\right)\right]^{-1}
$$

et

$U_{1}=U_{\mathrm{t}}\left[1+h_{\mathrm{pa}} U_{\mathrm{t}} /\left(h_{\mathrm{rpv}} h_{\mathrm{va}}+h_{\mathrm{pa}} h_{\mathrm{rpv}}+h_{\mathrm{va}} h_{\mathrm{pa}}\right)\right]^{-1}$

$U_{1}$ étant le coefficient de pertes thermiques du verre extérieur.

Les quantités $h_{\mathrm{r}}$ et $h$ désignent les coefficients d'échanges radiatifs linéarisés et d'échanges convectifs, les indices $\mathrm{p}, \mathrm{v}$ et a se référant à l'absorbeur, à la vitre, et à l'ambiance extérieure.

La procédure numérique pour calculer le rendement du capteur consiste à partir d'une température $T_{\mathrm{v}}$ et $T_{\mathrm{p}}$ d'essai, à écrire le bilan thermique et en déduire de nouvelles températures de vitre et d'absorbeur, en itérant jusqu'à convergence des valeurs. Ce travail a été fait sur un ordinateur Solar 16 en faisant varier divers paramètres physiques comme l'épaisseur de la lame d'air, le débit d'air, la nature de la couverture transparente, le rayonnement incident, la température extérieure et la température de reprise du capteur. L'examen des résultats montre que, pour obtenir un échauffement de l'air allant de $10^{\circ}$ à $20^{\circ} \mathrm{C}$ au-dessus d'une température de reprise voisine de $30^{\circ} \mathrm{C}$, le débit optimum est d'environ $30 \mathrm{~kg} / \mathrm{h} / \mathrm{m}^{2}$, la lame d'air idéale voisine de $2,5 \mathrm{~cm}$. Dans ces conditions, le rendement dépend du rayonnement incident et de la nature de la couverture transparente d'une façon qui est schématisée dans les figures 4 et 5 . On constate que dans le cas d'une simple vitre, le rendement, nul pour un rayonnement inférieur à $250 \mathrm{~W} / \mathrm{m}^{2}$, atteint $30 \%$ pour un fort rayonnement $\left(690 \mathrm{~W} / \mathrm{m}^{2}\right)$, l'échauffement de l'air n'excédant pas $15^{\circ} \mathrm{C}$. En revanche, une double vitre permet d'atteindre 35 à $55 \%$ de rendement avec un échauffement allant de $8^{\circ}$ à $35^{\circ} \mathrm{C}$ suivant l'insolation.

3.3 ETUDE EXPÉRIMENTALE DES ÉCHANGES THERMIQUES AU NIVEAU DU STOCKAGE. - Le modèle mathématique décrivant le transfert de chaleur à travers un milieu diphasique se complique quand la géométrie du volume de stockage devient arbitraire. Aussi l'étude de diverses formes d'éléments de stockage a-t-elle été faite expérimentalement.

Un banc d'essai a été construit dans lequel un élément de stockage à chaleur latente est soumis sur une face à un flux d'air chaud à diverses températures et vitesse fixe tandis que son autre face restitue de la chaleur à une ambiance calme à température fixe.

Deux types d'éléments de stockage très différents ont été expérimentés : dans le premier, le matériau à chaleur latente est enfermé dans un cylindre de PVC de $8 \mathrm{~cm}$ de diamètre dont une face est léchée par l'air chaud, et l'autre face noyée dans une paroi de plâtre qui restitue la chaleur à l'ambiance, les ponts thermiques étant soigneusement évités. Les mesures ont été faites avec une vitesse d'air de $2 \mathrm{~m} / \mathrm{s}$ des températures d'air chaud allant de $34^{\circ} \mathrm{C}$ à $77^{\circ} \mathrm{C}$ et une température d'ambiance de $17^{\circ} \mathrm{C}$. La figure 6 montre la fraction de matériau fondu en fonction du temps et de la température et justifie l'intérêt qu'il 


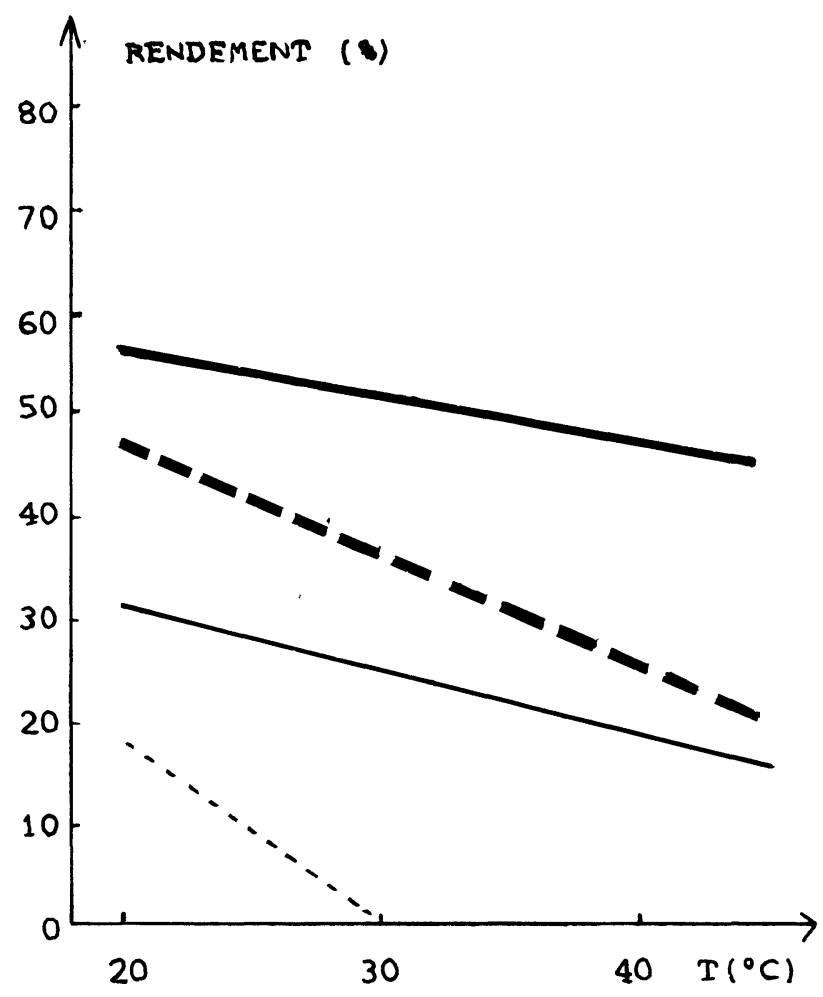

Fig. 4. - Résultat de modélisation du capteur à air en convection forcée ; trait interrompu pour un apport solaire faible $\left(250 \mathrm{~W} / \mathrm{m}^{2}\right)$; trait continu pour fort rayonnement $\left(690 \mathrm{~W} / \mathrm{m}^{2}\right)$; tracé fin pour une simple vitre, tracé épais pour une double vitre. Le rendement est représenté en fonction de la température d'entrée au capteur.

[Numerical simulation of the front air collector with forced convection; dashed lines for weak insolation $\left(250 \mathrm{~W} / \mathrm{m}^{2}\right)$, full lines for strong insolation $\left(690 \mathrm{~W} / \mathrm{m}^{2}\right)$; thin lines for single glazing, thick lines for double glazing. $T$ is the incoming air temperature.]

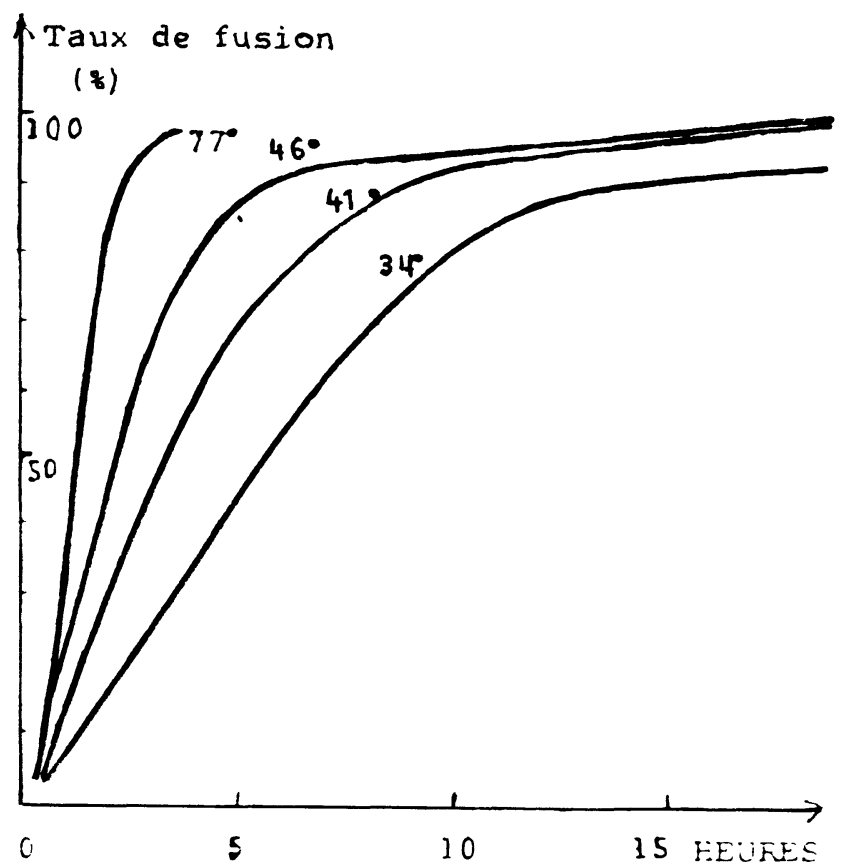

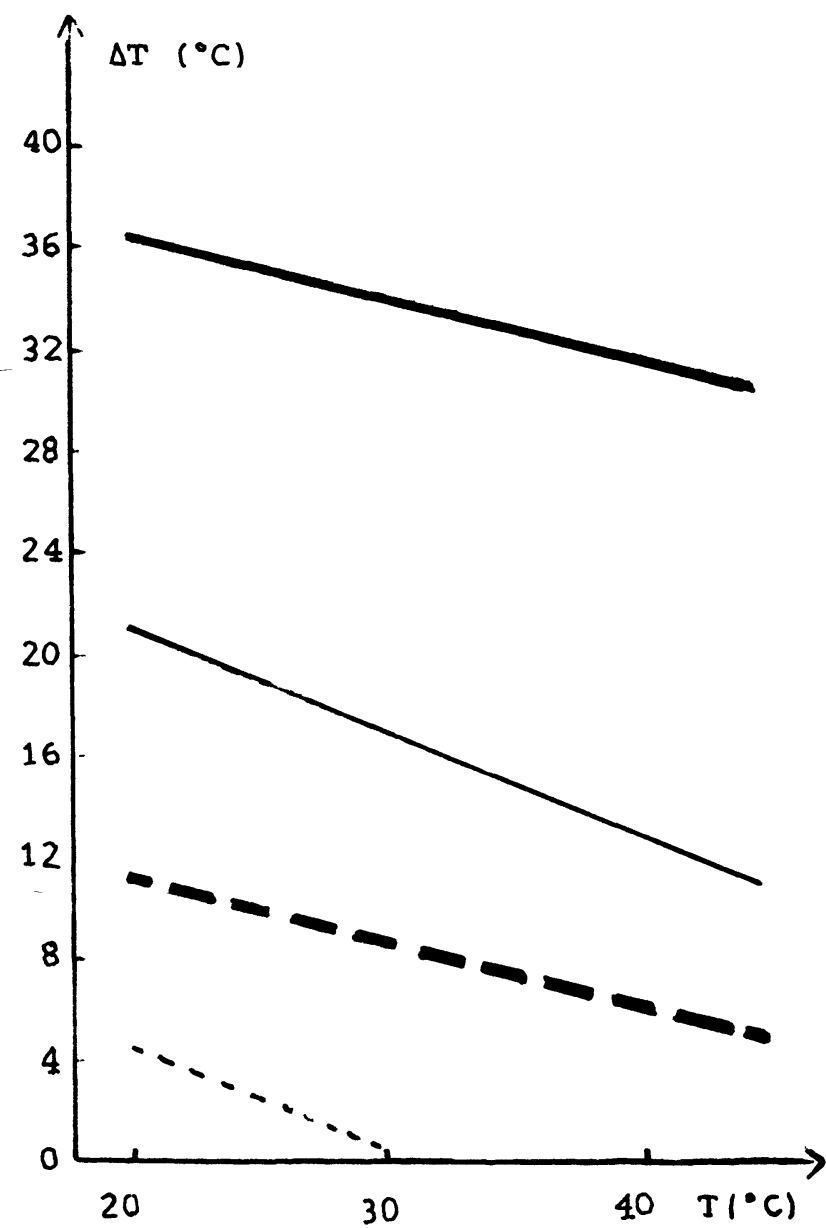

Fig. 5. - Mêmes conventions qu'en figure 4. L'échauffement de l'air dans le capteur est tracé en fonction de la température d'entrée.

[Temperature increase of the air delivered by the collector as a function of incoming air temperature. Drawing conventions same as in figure 4.]

peut $\mathrm{y}$ avoir à produire un air même tiède $\left(34^{\circ} \mathrm{C}\right)$ sur la face avant d'un mur diode par temps peu ensoleillé. La figure 7 indique la répartition des températures dans le stockage au cours du temps lors d'un régime alterné de $5 \mathrm{~h}$ d'air chaud à $46^{\circ}$ suivi de $19 \mathrm{~h}$ sans apports. On constate en particulier que la face du mur qui restitue à l'ambiance ne ressent plus les fluctuations incidentes que sous une forme très atténuée et permet donc un apport thermique très régulier à l'ambiance.

Le deuxième type d'élément de stockage étudié est un parpaing de béton creux dont les alvéoles

Fig. 6. - Résultats expérimentaux sur le transfert thermique vers le matériau à chaleur latente stocké en tubes PVC. Le taux de fusion est porté en fonction du temps pour diverses températures d'air du capteur.

[Experimental heat transfers observed in a cylindrical latent heat storage. The melting rate is represented for various collector air temperatures.] 


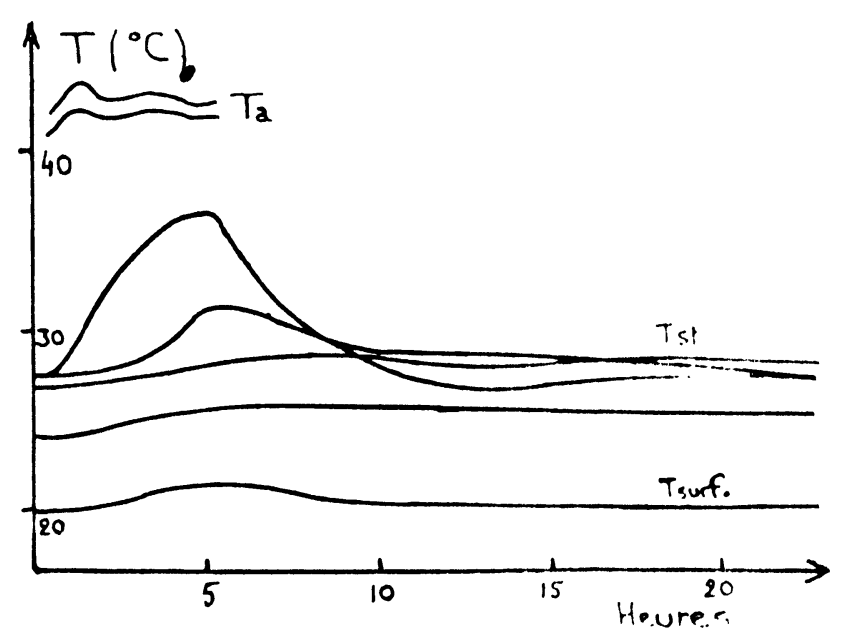

Fig. 7. - Simulation d'un régime journalier répétitif de $5 \mathrm{~h}$ d'apport d'air chaud à $46^{\circ}$ (courbes $T_{\mathrm{a}}$ ) suivies de $19 \mathrm{~h}$ de refroidissement. Les courbes $T_{4}$ montrent l'évolution de la température dans des zones successives du stockage cylindrique. La restitution vers le volume habitable est commandée par la température de surface $T_{\text {sur }}$.

[Experimental simulation of successive daily cycles with 5 hours solar gains at $46^{\circ} \mathrm{C}$ (curves $T_{\mathrm{a}}$ ) followed by 19 hours of cooling. Curves $T_{\mathrm{st}}$ refer to various positions in the cylindrical storage: curve $T_{\text {surf }}$ shows the surface temperature of the wall which commands the heat transfer to the living space.]

étanchéifiées ont été remplies de matériau à chaleur latente, une circulation d'air étant aménagée à travers les alvéoles. L'avantage d'un tel élément de stockage est qu'il se prête à une utilisation simple dans le bâtiment. Son défaut est d'une part l'existence de ponts thermiques vers la face restituant la chaleur, à cause de la conductivité du béton, et c'est ce que montre la figure 8 où l'on voit la température de paroi suivre de trop près la température de l'air chaud; d'autre part la fraction non négligeable de chaleur sensible qui intervient dans le stockage thermique global (près de $40 \%$ ). C'est néanmoins ce type de parpaing qui a servi à la construction d'un

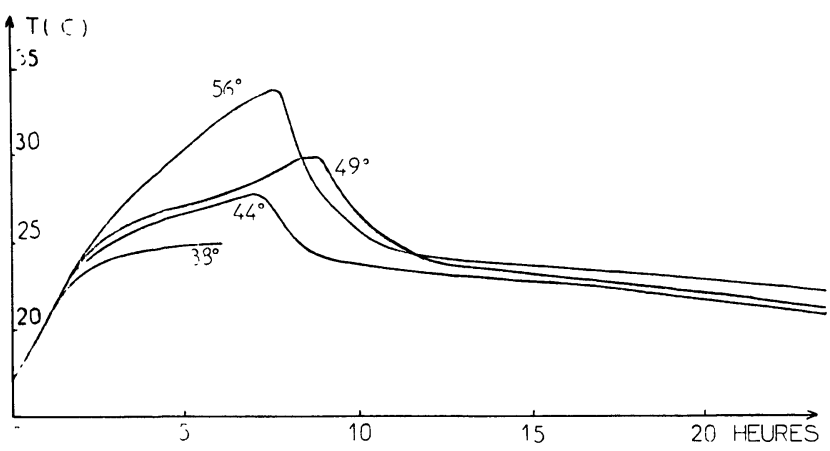

Fig. 8. - Cycle thermique journalier d'un parpaing de beton rempli de chliarolithe. La période diurne s'étale ici sur $6 \mathrm{~h} 45$ avec diverses températures d'air chaud. Les courbes donnent l'évolution de la température moyenne de surface au cours du temps.

[Daily thermal cycle of a concrete block filled with chliarolithe. The heating period lasts $6 \mathrm{~h} 45$ with various air temperatures. The average surface temperature of the block is plotted versus time.] mur diode de taille normale pour une campagne de mesure en conditions réelles.

3.4 COMPORTEMENT D'UN MUR DIODE EN CONDITIONS RÉELLES. - Une cellule expérimentale, visible en figure 9, a été construite sur le terrain de l'Observatoire de Nice. Un mur diode de $3,5 \mathrm{~m}^{2}$ et de $2,2 \mathrm{~m}$ de haut, construit en parpaings de ciment à alvéoles remplies de chliarolithe, sert de paroi chauffante à un local de $4 \mathrm{~m}^{3}$ modérément isolé. Le capteur à air

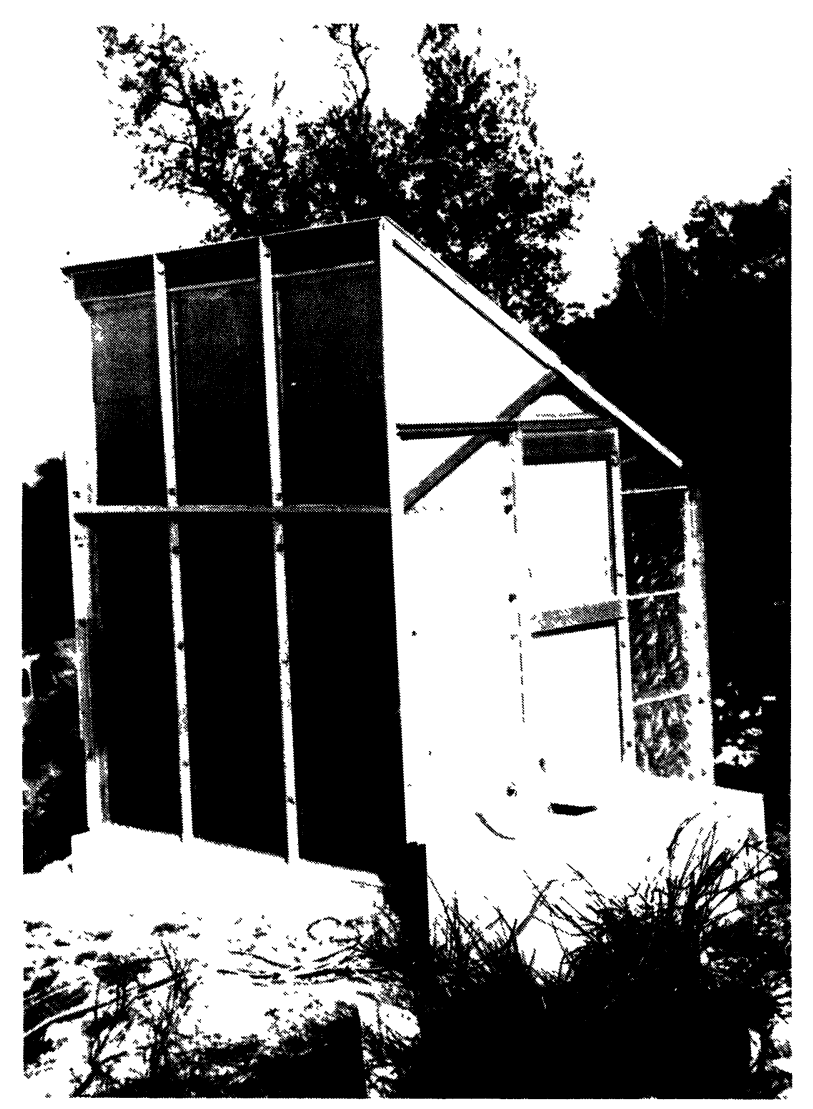

Fig. 9. - Cellule test construite pour l'étude en conditions naturelles d'un mur diode en parpaings de béton à chaleur latente.

[Test cell constructed in Nice to study under natural conditions a concrete block latent heat diode wall.]

de la face avant possède une double vitre et l'absorbeur est une tôle d'aluminium déployé noircie. Un ventilateur de $90 \mathrm{~W}$ assure une circulation forcée de $150 \mathrm{~kg}$ d'air par heure entre le capteur et le stockage pendant la journée. Une description plus complète du mur diode et de son comportement peut être trouvée en [9]. Le rendement du capteur à air au cours d'une journée à ensoleillement variable est donné en figure 10 : ce rendement est une fonction croissante du rayonnement incident et varie de $30 \%$ à $60 \%$ pour une température de reprise d'air de $29^{\circ} \mathrm{C}$ environ. Ce résultat est légèrement supérieur aux prédictions du modèle décrit en 3.2 , mais le gain provient de la surface d'échange accrue offerte par l'absorbeur 


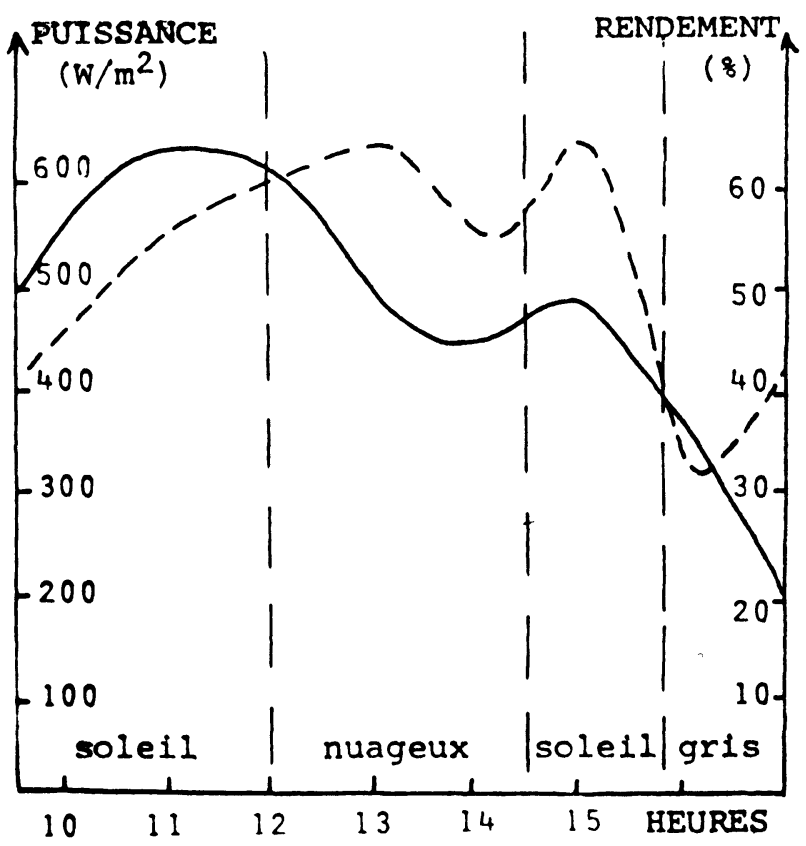

Fig. 10. - Rayonnement solaire reçu par le mur diode (trait plein), et rendement de captage observé au cours d'une journée type, (trait interrompu).

[Solar radiation (full line) incident on the diode wall and collector efficiency (dotted line) during a typical day.]

en aluminium déployé. La figure 11 montre l'évolution des températures au cours d'une journée typique d'hiver en région niçoise. L'air fourni par le capteur atteint une température de plus de $50^{\circ} \mathrm{C}$, soit un échauffement d'une vingtaine de degrés par rapport au niveau de reprise. Les ponts thermiques dans les parpaings de béton sont responsables d'une élévation un peu trop sensible de la température du local dans la journée. En revanche la stabilisation de la température nocturne du local est satisfaisante. Sur une période de 30 jours consécutifs, avec des températures

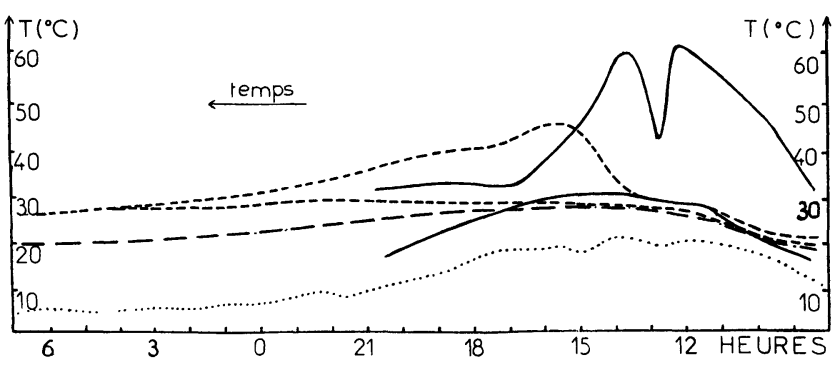

Fig. 11. - Températures observées dans la cellule test du,mur diode au cours d'une journée type ; en traits pleins, la température d'air à l'entrée et au milieu du stockage : en traits interrompus la température de surface du mur au niveau supérieur et au niveau médian; en trait mixte, la température du volume habitable; en trait pointillé la température extérieure.

[Observed temperatures in a test cell submitting the diode wall to the daily solar radiation. Air temperatures at the top and at the middle of the storage in full lines; surface temperatures of the wall at top and middle levels in dashed lines; ambiant temperature in mixed line, outside temperature in dotted line.] nocturnes de l'ordre de $2,5^{\circ} \mathrm{C}$, la température du local expérimental a été maintenue régulièrement au-dessus de $18^{\circ} \mathrm{C}$, bien que son taux de déperdition soit de $4 \mathrm{~W} / \mathrm{m}^{3}{ }^{\circ} \mathrm{C}$. L'enseignement tiré de cette expérience pratique permet de situer les points faibles et de prévoir les améliorations possibles : l'isolant de polystyrène qui sépare le capteur du stockage, diffusant dans l'infrarouge, laisse un taux de pertes radiatives trop élevé; il conviendrait de lui substituer un matériau du genre mousse de verre, qui offrirait de plus une meilleure tenue à la chaleur; les parpaings de béton offriraient une meilleure homogénéité de température si la circulation d'air se faisait plus loin de la face de restitution. Il suffit pour cela de choisir des modèles à deux rangées d'alvéoles, une rangée étant consacrée à la circulation de l'air. Des mesures ont montré que les ponts thermiques sont ainsi nettement plus faibles.

Il est possible enfin de faire appel à des parpaings de béton allégé (argile expansée et ciment) dans lesquels l'effet de filtre thermique de la chaleur latente serait plus accentué, la fraction de chaleur sensible dans la chaleur totale accumulée étant plus faible d'un facteur 2 (le supplément de résistance thermique à travers les parois de $15 \mathrm{~mm}$ de béton allégé ne constitue pas un handicap insurmontable).

4. Mur diode en thermosiphon. - Le mur diode en convection forcée décrit dans le chapitre précédent ne peut pas prétendre être une paroi solaire passive au même titre qu'un mur Trombe. Il existe en effet un ventilateur en fonctionnement diurne. Il existe pourtant des situations où un fonctionnement entièrement passif et autonome serait souhaitable : par exemple lors de la climatisation de locaux en altitude, en l'absence d'électricité, ou de la mise hors gel d'habitations inoccupées. Aussi est-il apparu nécessaire d'examiner des conditions dans lesquelles un mur diode pourrait fonctionner en convection naturelle.

\subsection{FONCTIONNEMENT D'UN CAPTEUR A AIR EN} CONVECTION NATURELLE. - Il convient d'examiner, dans un premier temps l'échauffement de l'air, son débit et le rendement de captage en l'absence de ventilateur, abstraction étant faite du circuit de refroidissement situé en arrière.

Les conditions réelles sont intermédiaires entre le cas de la convection naturelle le long d'une plaque verticale chauffée à flux constant et le cas de la convection naturelle le long d'une plaque chauffée à température constante. En effet le flux que peut transmettre la plaque à l'air est majoré par le rayonnement solaire incident, ce qui exclut une température d'absorbeur constante dans la partie basse du capteur; d'autre part, les pertes croissant avec la température de l'absorbeur, le flux convecté à l'air ne peut que décroître, la température de l'absorbeur étant majorée par une température limite où les pertes égalent les apports. Pour simplifier cette étude, nous supposerons que la couverture transparente du 
capteur ne perturbe pas les couches limites thermique et dynamique qui se forment au contact de l'absorbeur; cela se traduira par une distance minimale à respecter suivant la hauteur totale du capteur. Nous supposerons de même que la température de cette couverture ne diffère pas sensiblement de la température d'entrée de l'air $T_{0}$ au bas du capteur de sorte qu'il ne se crée pas de circulation parasite au contact de celle-ci. Les constantes physiques de l'air sont prises à $320 \mathrm{~K}$. Dans ces conditions, la description de la convection au contact de l'absorbeur à flux constant $\varphi$ prend la forme (en régime laminaire) :

où

$$
\begin{gathered}
T_{\mathrm{p}}(x)-T_{0}=0,966 \varphi^{4 / 5} x^{1 / 5} \\
u(x, y)=0,082 x^{3 / 5} \varphi^{2 / 5} F_{\iota p}^{\prime}(\eta) \\
T(x, y)-T_{0}=\left(T_{\mathrm{p}}(x)-T_{0}\right) \theta_{\varphi p}(\eta)
\end{gathered}
$$

$$
\eta=57,7 y x^{-1 / 5} \varphi^{1 / 5}
$$

et $F_{\varphi}^{\prime}(\eta)$ et $\theta_{\varphi}(\eta)$ sont les fonctions tabulées par Gregg et Sparrow [10].

$x$ désigne l'altitude le long du capteur, $y$ la distance à l'absorbeur.

On peut définir une épaisseur de couche limite $\delta$ comme 4 fois la distance $y_{\max }$ où la vitesse $u$ atteint son maximum à $x$ fixé; alors

$$
\delta(x)=6,58 \times 10^{-2} \varphi^{-1 / 5} x^{1 / 5} .
$$

Dans les applications numériques étudiées ici, une paramétrisation simple a été adoptée pour les fonctions $\theta_{\varphi}(\eta)$ et $F_{\varphi}^{\prime}(\eta)$ :

et

$$
\theta_{\varphi}(\eta)=\left[\frac{6-1,92 \eta}{6+\eta}\right]^{1,61} \quad \text { pour } \eta<3,13
$$

$$
\theta_{\varphi}(\eta)=0 \quad \text { pour } \eta \geqslant 3,13
$$

$F_{\varphi}^{\prime}(\eta)=0,576 \eta^{0,857}(1-0,270 \eta)^{2,544}$ pour $\eta<3,70$ et

$F_{\varphi}^{\prime}(\eta)=0$

pour $\eta \geqslant 3,70$.

La description de la convection naturelle le long d'une plaque chauffée à $T_{\mathrm{p}}=$ Cte prend la forme suivante [11] (en régime laminaire) :

où

$$
\begin{gathered}
\varphi(x)=1,08\left(T_{\mathrm{p}}-T_{0}\right)^{5 / 4} x^{-1,4} \\
u(x, y)=0,25 x^{1 / 2}\left(T_{\mathrm{p}}-T_{0}\right)^{1 / 2} F_{\mathrm{T}}^{\prime}(\eta) \\
T(x, y)-T_{0}=\left(T_{\mathrm{p}}-T_{0}\right) \theta_{\mathrm{T}}(\eta)
\end{gathered}
$$

$$
\eta=70,7\left(T_{\mathrm{p}}-T_{0}\right)^{1 / 4} x^{-1 / 4} y
$$

$\varphi_{\mathrm{T}}(x)$ étant le flux convecté à l'air au niveau $x$.

On peut définir comme précédemment une épaisseur de couche limite

$$
\delta_{\mathrm{T}}(x)=0,056 x^{1 / 4}\left(T_{\mathrm{p}}-T_{0}\right)^{-1 / 4} .
$$

Le flux moyen par $\mathrm{m}^{2}$ convecté à l'air dans un capteur de hauteur $H$ vaut alors

$$
Q_{\mathrm{H}}=1,44\left(T_{\mathrm{p}}-T_{0}\right)^{5 / 4} H^{-1 / 4}
$$

tandis que l'on avait évidemment $Q_{\mathrm{H}}=\varphi=$ Cte dans le cas précédent.

Une paramétrisation analogue des fonctions $\theta_{\mathrm{T}}(\eta)$ et $F_{\mathrm{T}}^{\prime}(\eta)$ a été utilisée

$$
\begin{array}{lll}
\theta_{\mathrm{T}}(\eta)=\left[\frac{6-1,5 \eta}{6+\eta}\right]^{1,55} & \text { pour } \eta<4 \\
\theta_{\mathrm{T}}(\eta)=0 & \text { pour } \eta \geqslant 4 \\
F_{\mathrm{T}}^{\prime}(\eta)=0,576 \eta^{0,857}(1-0,252 \eta)^{2,544} & \text { pour } \eta<3,97 \\
F_{\mathrm{T}}^{\prime}(\eta)=0 & \text { pour } \eta \geqslant 3,97 .
\end{array}
$$

A l'aide de ces modèles laminaires, les comportements extrêmes au tout début du capteur (flux constant) et tout en haut du capteur ( $T_{\mathrm{p}}$ constant) ont été étudiés, dans le cadre des conditions physiques suivantes.

Rayonnement incident : $740 \mathrm{~W} / \mathrm{m}^{2}$; rayonnement absorbé $666 \mathrm{~W} / \mathrm{m}^{2}$; température extérieure $T_{\mathrm{e}}=10^{\circ} \mathrm{C}$; température de ciel $T_{\mathrm{c}}=0^{\circ} \mathrm{C}$; température de reprise d'air $T_{0}=33^{\circ} \mathrm{C}$; coefficient d'échange convectif au niveau d'une simple vitre $h_{\mathrm{v}}=5,5 \mathrm{~W} / \mathrm{m}^{2}{ }^{\circ} \mathrm{C}$. Le modèle à flux constant $\varphi$ est alors appliqué avec une valeur calculée $\varphi=500 \mathrm{~W} / \mathrm{m}^{2}$ qui correspond à une vitre à $20^{\circ} \mathrm{C}$ en bas de capteur. Le modèle à

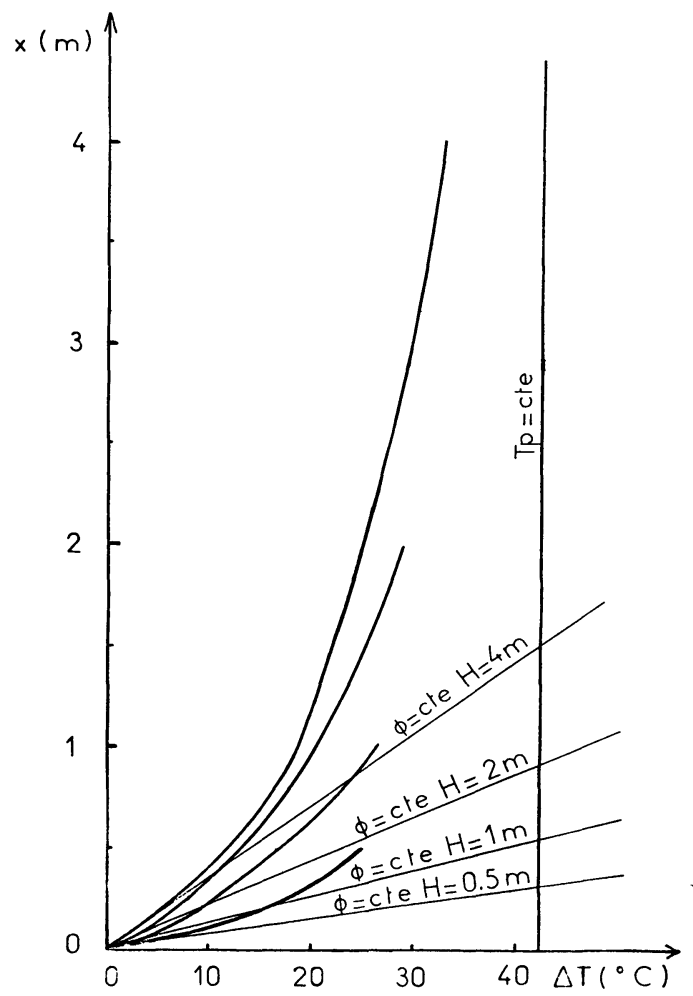

Fig. 12. - Résultat de la modélisation de l'échauffement de l'air dans un capteur en convection naturelle laminaire, en fonction de la hauteur $H$ globale du capteur et de l'altitude dans le capteur. Les droites représentent le comportement limite à température $T_{\mathrm{p}}$ constante ou à flux $\varphi$ constant.

[Numerical simulation of the temperature rise of the air in a collector operating in a natural convection mode (laminar); results for collectors of various heights $H$ are indicated. Straight lines correspond to the behavior at constant plate temperature or constant convective flux from the plate.] 
température constante est appliqué avec une température limite de plaque $T_{\mathrm{p}}=115,5^{\circ} \mathrm{C}$ qui correspond à une vitre à $60^{\circ} \mathrm{C}$. La distance entre vitre et absorbeur est prise égale à $\delta_{\mathrm{T}}(H)$. Dans ces conditions les valeurs extrêmes de l'échauffement de l'air sont données en figure 11 par la droite verticale en haut de capteur et les droites inclinées en bas du capteur. Pour connaître le comportement intermédiaire, un calcul de type perturbatif a été effectué où les expressions du modèle à $T_{\mathrm{p}}=$ Cte sont supposées s'appliquer et où la température de l'absorbeur (et donc de la vitre) est modifiée pour rétablir un bilan équilibré à une hauteur intermédiaire. Ce calcul perturbatif se traduit par des courbes, visibles dans la figure 12 , qui tendent à relier les comportements extrêmes. On voit que la température moyenne de l'air produit par le capteur croît très lentement pour des capteurs de hauteurs croissantes $\left(25^{\circ} \mathrm{C}\right.$ pour $H=0,5,33^{\circ} \mathrm{C}$ pour $\left.H=4 \mathrm{~m}\right)$. D'autre part une hauteur de $0,5 \mathrm{~m}$ suffit déjà largement à produire un échauffement adapté au stockage : on ne peut que faire chuter le rendement de captage avec des hauteurs plus grandes. A partir des températures de vitre, on peut estimer que ce rendement est voisin de $50 \%$ pour une hauteur de $0,5 \mathrm{~m}$, il n'est plus que de $20 \%$ pour une hauteur de $4 \mathrm{~m}$, en négligeant l'apport convectif de la vitre à l'air du capteur et le passage en mode turbulent.

4.2 L'étape suivante dans la description du fonctionnement du mur diode en convection naturelle consiste à inclure l'influence du circuit de refroidissement sur le circuit de captage. La première remarque est qu'il n'est pas possible d'avoir un régime permanent de transfert de chaleur entre capteur et stockeur si les deux processus de réchauffement et de refroidissement sont symétriques par rapport à un plan vertical : l'effet de thermosiphon s'annule après un régime transitoire. Il faut donc envisager une des deux géométries indiquées en figure 13. Dans l'une, la zone de stockage reste dans le plan vertical mais se situe au-dessus du capteur, ce qui suggère un assemblage de murs diodes de faible hauteur imbriqués les uns dans les autres; dans l'autre, le capteur débouche sur un stockage en dalle horizontale situé au niveau supérieur. Dans ces deux cas, la perte de charge du circuit de stockage provoque une modification du régime de convection naturelle dans le capteur : un ralentissement de la circulation et par suite un échauffement plus grand de l'air. Le rende-

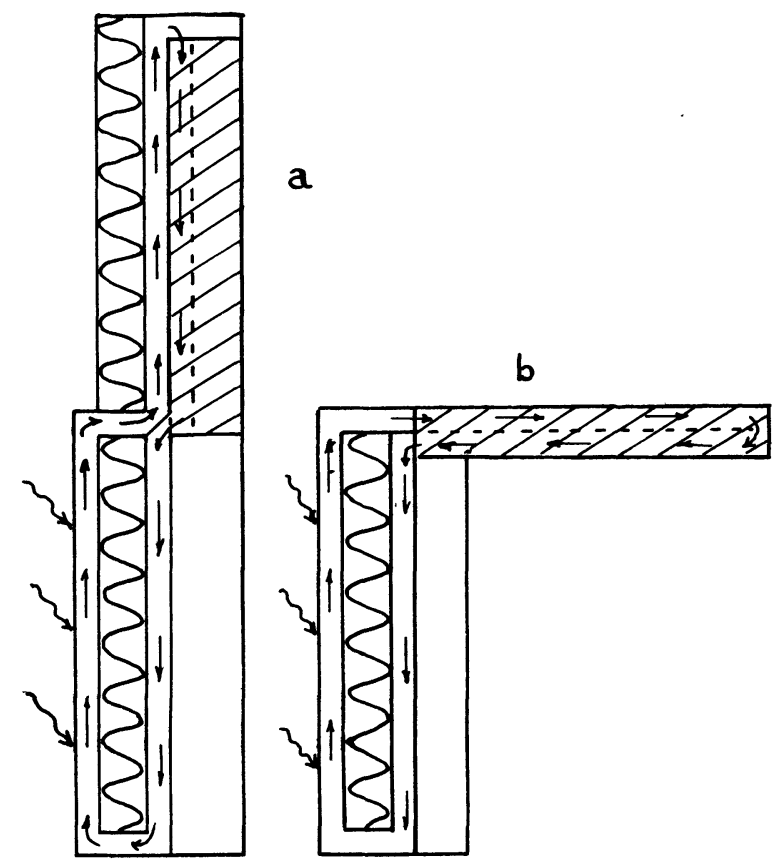

Fig. 13. - Géométries possibles pour un mur diode fonctionnant en thermosiphon. En figure $13 a$, une disposition permettant d'imbriquer des murs diodes sur un plan vertical ; en figure $13 b$ une disposition mixte, captage vertical, stockage en dalle.

[Possible geometries for a thermosiphon diode wall. The configuration in figure $13 a$ can be extrapolated for successive diode panels on a vertical plane; in the configuration of figure $13 b$, the storage takes place in a floor or a ceiling.]

ment s'en trouve abaissé d'autant. Si l'on accepte une vitesse moyenne moitié moindre, l'échauffement et les pertes seront accrus de $33 \%$ dans le cas du capteur de $0,5 \mathrm{~m}$ de haut et le rendement ne sera plus que de $33 \%$. Mais le supplément de poussée d'Archimède et la réduction de la vitesse permettent alors de venir à bout d'une perte de charge dans le stockage sensiblement 4 fois supérieure à celle du circuit avant, ce qui permet d'améliorer les échanges au sein du stockage.

En conclusion, le fonctionnement d'un mur diode en convection naturelle est bien plus contraignant qu'en convection forcée, mais il est possible de parvenir à un compromis rendant ce système solaire passif au prix d'une chute du rendement et d'une géométrie moins souple. 


\section{Bibliographie}

[1] Chliarolithe, matériau de stockage par chaleur latente à base de chlorure de calcium hexahydrate; brevet Anvar CNRS no 7913296.

[2] Sel de Glauber stabilisé. Divers brevets dont M. Telkes US Patent 3986969 de 1976.

[3] Тromвe, F., Maisons solaires. Techniques de l'ingénieur (3) $1974 \mathrm{C}$

[4] Stargardt, W. and Buckley, S., "An economic analysis of thermic diode solar panels». ASME Paper 76-WA/ SOP-7 1976.

[5] Trombe, F., Dans Applications thermiques de l'énergie solaire dans le domaine de la recherche et l'industrie. Montlouis Juin 58. Paris CNRS 1976.

[6] Bourdeau, L. and Jaffrin, A., "Phase change collector wall versus water collector wall». Proceedings of the
International Symposium on Solar Energy. Cairo Egypt, June 1978.

[7] Duffie, J. A., Beckman, W. A., Solar energy thermal processes (J. Wiley and Sons) 1974.

[8] Moisan, A., "Mur diode : modélisation mathématique et performances annuelles ", rapport UNESCO 1978.

[9] Bourdeau, L., JAFFrin, A., "Actual performance of a latent heat diode wall ». Proceedings of Izmir International Symposium II on Solar Energy Fundamentals and applications. Izmir Turkey, Aug. 1979.

[10] GregG, J. L. and Sparrow, E. M., "Low Prandtl number free convection", Z. Angew. Math. Phys. IX a Fasc. 4 (1958).

[11] Schmidt, E. and Beckman, Techn. Mech. Thermodyn. Bd. 1, $\mathrm{Nr} 10$ (1930) und $\mathrm{Nr} 11$ (1930). 